\title{
Homology of Spaces of Directed Paths in Euclidean Pattern Spaces
}

\author{
Roy Meshulam* $\quad$ Martin Raussen ${ }^{\dagger}$
}

October 11, 2018

In memory of Jirka Matoušek

\begin{abstract}
Let $\mathcal{F}$ be a family of subsets of $\{1, \ldots, n\}$ and let

$$
Y_{\mathcal{F}}=\bigcup_{F \in \mathcal{F}}\left\{\left(x_{1}, \ldots, x_{n}\right) \in \mathbb{R}^{n}: x_{i} \in \mathbb{Z} \text { for all } i \in F\right\}
$$

Let $X_{\mathcal{F}}=\mathbb{R}^{n} \backslash Y_{\mathcal{F}}$. For a vector of positive integers $\mathbf{k}=\left(k_{1}, \ldots, k_{n}\right)$ let $\vec{P}\left(X_{\mathcal{F}}\right)_{\mathbf{0}}^{\mathbf{k}+\mathbf{1}}$ denote the space of monotone paths from $\mathbf{0}=(0, \ldots, 0)$ to $\mathbf{k}+\mathbf{1}=\left(k_{1}+1, \ldots, k_{n}+1\right)$ whose interior is contained in $X_{\mathcal{F}}$. The path spaces $\vec{P}\left(X_{\mathcal{F}}\right)_{\mathbf{0}}^{\mathbf{k}+\mathbf{1}}$ appear as natural examples in the study of Dijkstra's PV-model for parallel computations in concurrency

We study the topology of $\vec{P}\left(X_{\mathcal{F}}\right)_{\mathbf{0}}^{\mathbf{k}+\mathbf{1}}$ by relating it to a subspace arrangement in a product of simplices. This, in particular, leads to a computation of the homology of $\vec{P}\left(X_{\mathcal{F}}\right)_{\mathbf{0}}^{\mathbf{k}+\mathbf{1}}$ in terms of certain order complexes associated with the hypergraph $\mathcal{F}$.
\end{abstract} theory.

\section{Introduction}

Concurrency theory in computer systems deals with properties of systems in which several computations are executing simultaneously and potentially interacting with each other. Among the many models suggested for the study of concurrency are the Higher Dimensional Automata (HDA) introduced by Pratt [11]. Those arise as cubical complexes in which individual cubes (of varying dimension) with directed paths on each of them, are glued together consistently. Compared to other concurrency models, HDA have the highest

*Department of Mathematics, Technion, Haifa 32000, Israel. e-mail: meshulam@math.technion.ac.il . Supported by ISF and GIF grants.

†Department of Mathematical Sciences, Aalborg University, Fredrik Bajersvej 7G, 9220 Aalborg Øst, Denmark. e-mail: raussen@math.aau.dk . Both authors acknowledge support from the ESF research networking programme ACAT. 


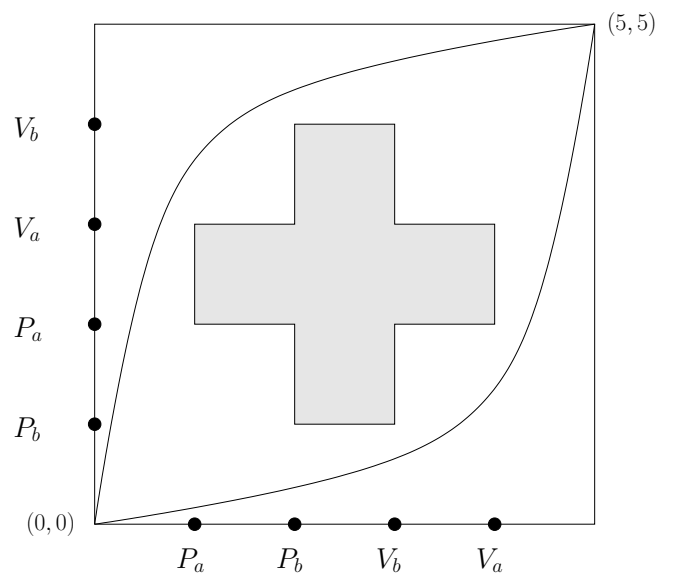

$$
\begin{aligned}
& T_{1}=P a \cdot P b \cdot V b \cdot V a \\
& T_{2}=P b \cdot P a \cdot V a \cdot V b \\
& \kappa(a)=\kappa(b)=1
\end{aligned}
$$

The trace space is homotopy equivalent to $S^{0}$

Figure 1: The Swiss flag example - two processes sharing two resources.

expressive power based on their ability to represent causal dependence [7]. On the other hand, only little is known in general about the topology of the space of directed paths of a general HDA [13].

A specific simple case of linear HDA's consists of the PV-model suggested by Dijkstra [4] back in the 1960's. In this model there are $m$ resources (e.g. shared memory sites) $a_{1}, \ldots, a_{m}$ with positive integer capacities $\kappa\left(a_{1}\right), \ldots, \kappa\left(a_{m}\right)$ where $\kappa\left(a_{i}\right)$ indicates the maximal number of processes that $a_{i}$ can serve at any given time, and $n$ linear processes $T_{1}, \ldots, T_{n}$ (without branchings or loops) that require access to these resources. Given a resource $a$ and a process $T$, denote by $P a$ and $V a$ the locking and respectively unlocking of $a$ by $T$. A process $T_{i}$ is specified by a sequence of locking and unlocking operations on the various resources in a certain order. Modeling each process $T_{i}$ as an ordered sequence of integer points on the interval $\left(0, k_{i}\right]$, one can view a legal execution of $\mathbf{T}=\left(T_{1}, \ldots, T_{n}\right)$ as a coordinate-wise nondecreasing continuous path from $\mathbf{0}=(0, \ldots, 0)$ to $\mathbf{k}+\mathbf{1}=\left(k_{1}+1, \ldots, k_{n}+1\right)$ that avoids a forbidden region determined by the processes and by the capacities of the resources. If two such paths are homotopic via a homotopy respecting the monotonicity condition then corresponding concurrent computations along the two paths have always the same result $([6,5])$.

Let $X_{\mathbf{T}, \kappa}$ denote the complement of the forbidden region in $\prod_{i=1}^{n}\left[0, k_{i}+1\right]$. The trace space $\vec{P}\left(X_{\mathbf{T}, \kappa}\right)_{\mathbf{0}}^{\mathbf{k}+\mathbf{1}}$ associated with the pair $(\mathbf{T}, \kappa)$ consists of all paths as above endowed with the compact-open topology. For example, for the two processes sharing two resources depicted in Figure 1, the forbidden region is the "Swiss Flag" and the trace space is homotopy equivalent to the two point space $S^{0}$. For an analysis of the PV spaces $X_{\mathbf{T}, \kappa}$ and their associated trace spaces $\vec{P}\left(X_{\mathbf{T}, \kappa}\right)_{\mathbf{0}}^{\mathbf{k}+\mathbf{1}}$, we refer to $[6,12,19,5]$.

In this paper we consider a special class of PV models in which the access and release of every resource happen without time delay. In this case, the forbidden region is a union of sets of the form $B \cap\left(K_{1} \times \cdots \times K_{n}\right)$, where $B$ is a fixed aligned box and each $K_{i}$ is either $\mathbb{Z}$ or $\mathbb{R}$. Our main result (see Theorem 1.3 below) is a formula for the Poincaré series 
of the trace spaces associated to such special PV models. We proceed with some formal definitions leading to the statement of Theorem 1.3.

Let $X$ be a subspace of $\mathbb{R}^{n}$. A continuous path $\mathbf{p}=\left(p_{1}, \ldots, p_{n}\right): I=[0,1] \rightarrow X \subset \mathbb{R}^{n}$ is called directed if all components $p_{i}: I \rightarrow \mathbb{R}$ are non-decreasing. For two points $y_{0}$ and $y_{1}$ in the closure of $X$, let $\vec{P}(X)_{\mathbf{y}_{0}}^{\mathbf{y}_{1}}$ be the space of all directed paths in $\bar{X}$ (endowed with the compact-open topology) starting at $\mathbf{y}_{0}$ and ending at $\mathbf{y}_{1}$ whose interior is contained in $X$.

Let $\mathbb{N}$ denote the non-negative integers and let $\mathbb{N}_{+}$denote the positive integers. Let $\mathbf{k}=\left(k_{1}, \ldots, k_{n}\right) \in \mathbb{N}_{+}^{n}$ be a fixed vector, and let $\mathbf{0}=(0, \ldots, 0), \mathbf{1}=(1, \ldots, 1), \mathbf{k}+\mathbf{1}=$ $\left(k_{1}+1, \ldots, k_{n}+1\right)$. In this paper we study the topology of $\vec{P}(X)_{\mathbf{0}}^{\mathbf{k}+\mathbf{1}}$ for spaces $X$ that are associated with the special PV programs described above. Let $\mathcal{F}$ be a family of subsets of $[n]=\{1, \ldots, n\}$ and let

$$
Y_{\mathcal{F}}=\bigcup_{F \in \mathcal{F}}\left\{\left(x_{1}, \ldots, x_{n}\right) \in \mathbb{R}^{n}: x_{i} \in \mathbb{Z} \text { for all } i \in F\right\}
$$

The Euclidean Pattern Space associated with $\mathcal{F}$ is defined by $X_{\mathcal{F}}=\mathbb{R}^{n} \backslash Y_{\mathcal{F}}$, with a corresponding Path Space $\vec{P}\left(X_{\mathcal{F}}\right)_{\mathbf{0}}^{\mathbf{k}+\mathbf{1}}$.

Example: If $\mathcal{F}$ consists of the single set $[n]$ then $X_{\mathcal{F}}=\mathbb{R}^{n} \backslash \mathbb{Z}^{n}$. Raussen and Ziemiański [14] investigated the path space $\vec{P}\left(\mathbb{R}^{n} \backslash \mathbb{Z}^{n}\right)_{\mathbf{0}}^{\mathbf{k}+\mathbf{1}}$ and determined its homology groups and its cohomology ring. Their result concerning homology is the following:

Theorem 1.1 (Raussen and Ziemiański [14]). For $n \geq 3$

$$
\tilde{H}_{\ell}\left(\vec{P}\left(\mathbb{R}^{n} \backslash \mathbb{Z}^{n}\right)_{\mathbf{0}}^{\mathbf{k}+\mathbf{1}}\right)= \begin{cases}\mathbb{Z}^{\prod_{i=1}^{n}\left(\begin{array}{c}
k_{i} \\
m
\end{array}\right)} & \ell=(n-2) m, m>0 \\
0 & \text { otherwise. }\end{cases}
$$

The Betti number $\prod_{i=1}^{n}\left(\begin{array}{c}k_{i} \\ m\end{array}\right)$ in (2) corresponds to the number of strictly increasing integer sequences of length $m$ strictly between $\mathbf{0}$ and $\mathbf{k}+\mathbf{1}$.

In this paper we consider $\vec{P}\left(X_{\mathcal{F}}\right)_{\mathbf{0}}^{\mathbf{k}+\mathbf{1}}$ for general $\mathcal{F}$. Without loss of generality we may assume that $\mathcal{F}$ is upward closed, i.e. if $F \in \mathcal{F}$ and $F \subset F^{\prime} \subset[n]$ then $F^{\prime} \in \mathcal{F}$. It will also be assumed that $|F| \geq 2$ for all $F \in \mathcal{F}$ (otherwise $\vec{P}\left(X_{\mathcal{F}}\right)_{\mathbf{0}}^{\mathbf{k}+\mathbf{1}}$ is empty). We first introduce some terminology.

\section{Definition 1.2.}

(i) A subset $\mathcal{G} \subset \mathcal{F}$ is a matching if $G \cap G^{\prime}=\emptyset$ for all $G \neq G^{\prime} \in \mathcal{G}$. Let $M(\mathcal{F})$ denote the family of all nonempty matchings of $\mathcal{F}$, with partial order $\preceq$ given by $\mathcal{G} \preceq \mathcal{G}^{\prime}$ if for every $G \in \mathcal{G}$ there exists a $G^{\prime} \in \mathcal{G}^{\prime}$ such that $G \subset G^{\prime}$. For $K \subset[n]$ let

$$
M(\mathcal{F})_{\preceq K}=\{\mathcal{G} \in M(\mathcal{F}): G \subset K \text { for all } G \in \mathcal{G}\}
$$

and let $M(\mathcal{F})_{\prec K}=M(\mathcal{F})_{\preceq}(K) \backslash\{\{K\}\}$. The order complex of $M(\mathcal{F})_{\prec K}$ is denoted by $\Delta\left(M(\mathcal{F})_{\prec K}\right)$. 
(ii) For a function $\mathbf{m}: \mathcal{F} \rightarrow \mathbb{N}$ let $T_{\mathcal{F}}(\mathbf{m})$ be the (simple undirected) graph on the vertex set $\cup_{F \in \mathcal{F}}\{F\} \times[\mathbf{m}(F)]$, where two vertices $(F, i) \neq\left(F^{\prime}, i^{\prime}\right)$ are connected by an edge if $F \cap F^{\prime} \neq \emptyset$.

(iii) An orientation of a simple undirected graph $G=(V, E)$ will be determined by a function $\alpha: E \rightarrow V^{2}$ that maps an edge $\{u, v\} \in E$ to either $(u, v)$ or $(v, u)$. An orientation is acyclic if the resulting directed graph does not contain directed cycles. Let $\mathfrak{A}(G)$ denote the set of acyclic orientations of $G$ and let $a(G)=|\mathfrak{A}(G)|$. By a result of Stanley [15], $a(G)$ can be computed by evaluating the chromatic polynomial of $G$ at -1 .

For $\mathbf{m}: \mathcal{F} \rightarrow \mathbb{N}$ let

$$
\begin{aligned}
b_{\mathcal{F}, \mathbf{k}}(\mathbf{m}) & =\frac{a\left(T_{\mathcal{F}}(\mathbf{m})\right)}{\prod_{F \in \mathcal{F}} \mathbf{m}(F) !} \prod_{i=1}^{n}\left(\begin{array}{c}
k_{i} \\
\sum_{F \ni i} \mathbf{m}(F)
\end{array}\right), \\
c_{\mathcal{F}}(\mathbf{m}) & =\sum_{F \in \mathcal{F}} \mathbf{m}(F)(|F|-2)+1 .
\end{aligned}
$$

The reduced Poincaré series of a space $Y$ over a field $\mathbb{K}$ is defined by

$$
f_{\mathbb{K}}(Y, t)=\sum_{i \geq 0} \operatorname{dim} \tilde{H}_{i-1}(Y ; \mathbb{K}) t^{i}
$$

Our main result is the following

\section{Theorem 1.3.}

(i) If $H_{*}\left(\Delta\left(M(\mathcal{F})_{\prec F}\right) ; \mathbb{Z}\right)$ is free for all $F \in \mathcal{F}$ then $H_{*}\left(\vec{P}\left(X_{\mathcal{F}}\right)_{\mathbf{0}}^{\mathbf{k}+\mathbf{1}} ; \mathbb{Z}\right)$ is free.

(ii) For any field $\mathbb{K}$

$$
f_{\mathbb{K}}\left(\vec{P}\left(X_{\mathcal{F}}\right)_{\mathbf{0}}^{\mathbf{k}+\mathbf{1}}, t\right)=\sum_{0 \neq \mathbf{m} \in \mathbb{N}^{\mathcal{F}}} b_{\mathcal{F}, \mathbf{k}}(\mathbf{m}) t^{c_{\mathcal{F}}(\mathbf{m})} \prod_{F \in \mathcal{F}} f_{\mathbb{K}}\left(\Delta\left(M(\mathcal{F})_{\prec F}\right), t^{-1}\right)^{\mathbf{m}(F)} .
$$

The paper is organized as follows: In Section 2 we describe a subspace arrangement $D_{\mathcal{F}}$ that is homotopy equivalent to $\vec{P}\left(X_{\mathcal{F}}\right)_{\mathbf{0}}^{\mathbf{k}+\mathbf{1}}$. In Section 3 we state Theorem 3.1 that describes the homotopy type of the Alexander dual of $D_{\mathcal{F}}$ and then use it to prove Theorem 1.3. The proof of Theorem 3.1 is given in Section 4 which constitutes the main technical part of the paper. In Section 5 we discuss several applications arising from particular cases of Theorem 1.3. The easy conclusions about (higher) connectivity of path spaces in Section 5.4 are probably the most notable ones for applications in concurrency theory. Some open problems are mentioned in Section 6.

\section{Directed Paths via Subspace Arrangements}

Spaces of directed paths in a PV-model have been shown to be homotopy equivalent to certain finite prod-simplicial complexes that make homology computations possible - at 
least in principle $[12,5]$. Unfortunately, these complexes grow very fast in dimension and size. Here we give an alternative description as complement of a subspace arrangement. Remark that directedness has the consequence that such an arrangement has to be considered as a subset of a product of simplices and not of Euclidean space; this is the reason why classical results are not immediately applicable.

Let $\mathcal{F}$ be an upward closed hypergraph on $[n]$ and let $\mathbf{k}=\left(k_{1}, \ldots, k_{n}\right) \in \mathbb{N}_{+}^{n}$. In this section we describe a model for $\vec{P}\left(X_{\mathcal{F}}\right)_{\mathbf{0}}^{\mathbf{k}+\mathbf{1}}$ up to homotopy equivalence.

\section{Definition 2.1.}

(i) For $k \geq 1$ let $\stackrel{\circ}{\Delta}_{k}$ denote the open $k$-simplex

$$
\stackrel{\circ}{\Delta}_{k}=\left\{\left(x_{1}, \ldots, x_{k}\right) \in \mathbb{R}^{k}: 0<x_{1}<\cdots<x_{k}<1\right\} .
$$

For $k=0$ let $\stackrel{\circ}{\Delta}_{0}$ denote the one point space $\{*\}$.

For $\mathbf{k}=\left(k_{1}, \ldots, k_{n}\right) \in \mathbb{N}_{+}^{n}$ let

$$
N=\sum_{i=1}^{n} k_{i},[\mathbf{k}]=\prod_{i=1}^{n}\left[k_{i}\right], \text { and } \stackrel{\circ}{\Delta}_{\mathbf{k}}=\prod_{i=1}^{n} \stackrel{\circ}{\Delta}_{k_{i}} \subset \mathbb{R}^{N}
$$

(ii) For $F \subset[n]$ let $\left[\mathbf{k}_{F}\right]=\prod_{i \in F}\left[k_{i}\right]$. For $\mathbf{j}=(\mathbf{j}(i))_{i \in F} \in\left[\mathbf{k}_{F}\right]$ and $F^{\prime} \subset F$, the restriction $(\mathbf{j})_{\mid F^{\prime}} \in\left[\mathbf{k}_{F^{\prime}}\right]$ of $\mathbf{j}$ to $F^{\prime}$ is given by $(\mathbf{j})_{\mid F^{\prime}}(i)=\mathbf{j}(i)$ for all $i \in F^{\prime}$. A partial sequence is a pair $(F, \mathbf{j})$ where $F \subset[n]$ and $\mathbf{j}=(\mathbf{j}(i))_{i \in F} \in\left[\mathbf{k}_{F}\right]$. Let $S_{\mathcal{F}}$ be the family of all partial sequences $(F, \mathbf{j})$ where $F \in \mathcal{F}$ and $\mathbf{j} \in\left[\mathbf{k}_{F}\right]$.

(iii) For a partial sequence $(F, \mathbf{j})$ let

$$
G_{(F, \mathbf{j})}=\left\{\left(x_{i 1}, \ldots, x_{i k_{i}}\right)_{i=1}^{n} \in \prod_{i=1}^{n}{\stackrel{\circ}{k_{i}}}_{x_{i \mathbf{j}(i)}}=x_{i^{\prime} \mathbf{j}\left(i^{\prime}\right)} \text { for all } i, i^{\prime} \in F\right\} .
$$

Let

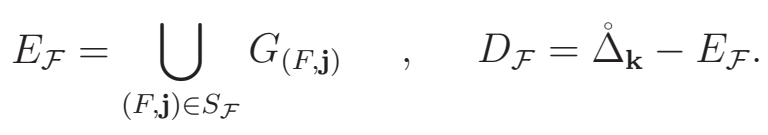

(iv) The one-point compactification of $\stackrel{\circ}{\mathbf{k}}_{\mathbf{k}}$ is given by

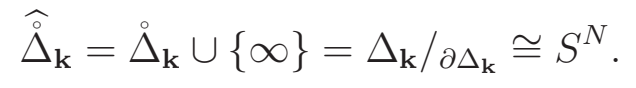

$\operatorname{For}(F, \mathbf{j}) \in S_{\mathcal{F}}$, the compactification of $G_{(F, \mathbf{j})}$ in $\widehat{\grave{\Delta}}_{\mathbf{k}}$ is given by $\Gamma_{(F, \mathbf{j})}=G_{(F, \mathbf{j})} \cup\{\infty\}$. The compactification of $E_{\mathcal{F}}$ in $\widehat{\vec{\Delta}}_{\mathbf{k}}$ is

$$
\widehat{E_{\mathcal{F}}}=E_{\mathcal{F}} \cup\{\infty\}
$$


Let $\vec{P}_{<}\left(X_{\mathcal{F}}\right)_{\mathbf{0}}^{\mathbf{k}+\mathbf{1}} \subset \vec{P}\left(X_{\mathcal{F}}\right)_{\mathbf{0}}^{\mathbf{k}+\mathbf{1}}$ denote the space of increasing directed paths $\mathbf{p}=\left(p_{1}, \ldots, p_{n}\right)$ : $I \rightarrow X_{\mathcal{F}} \subset \mathbf{R}^{n}$ characterized by $t<t^{\prime} \Rightarrow p_{i}(t)<p_{i}\left(t^{\prime}\right)$ (instead of $\leq$ ) for all $i$. Remark that every component $p_{i}$ is a homeomorphism of the unit interval.

A correspondence between the space $D_{\mathcal{F}}$ from Definition 2.1(iii) and this path space $\vec{P}_{<}\left(X_{\mathcal{F}}\right)_{0}^{\mathbf{k}+\mathbf{1}}$ and may be established as follows: For every $k \in \mathbb{N}_{+}$and $\mathbf{x}=\left(x_{1}, \ldots, x_{k}\right) \in \stackrel{\diamond}{\Delta}_{k}$ let $p_{\mathbf{x}}: I \rightarrow[0, k+1]$ denote the (directed) path with $p_{\mathbf{x}}(0)=0, p_{\mathbf{x}}(1)=k+1, p_{x}\left(x_{i}\right)=$ $i, 1 \leq i \leq k$, and connected by line segments inbetween. For every $\mathbf{0}<\mathbf{k} \in \mathbb{N}_{+}^{n}$ and every $\mathbf{x}=\left(\mathbf{x}_{1}, \ldots \mathbf{x}_{n}\right) \in \stackrel{\circ}{\mathbf{\Delta}}_{\mathbf{k}}($ cf. 6$)$, let $\mathbf{p}(\mathbf{x})(t)=\left(p_{\mathbf{x}_{1}}(t), \ldots, p_{\mathbf{x}_{n}}(t)\right)$. This recipe defines a continuous map $P: \stackrel{\triangle}{\Delta}_{\mathbf{k}} \rightarrow \vec{P}_{<}\left(\mathbf{R}^{n}\right)_{\mathbf{0}}^{\mathbf{k}+\mathbf{1}}$ that restricts to a map $P_{\mathcal{F}}^{<}: D_{\mathcal{F}} \rightarrow \vec{P}_{<}\left(X_{\mathcal{F}}\right)_{\mathbf{0}}^{\mathbf{k}+\mathbf{1}}$ : For $\mathbf{x}=\left(\mathbf{x}_{1}, \ldots, \mathbf{x}_{n}\right) \in \stackrel{\circ}{\Delta}_{\mathbf{k}}$ and $\mathbf{x}_{i}=\left(x_{i 1}, \ldots, x_{i k_{i}}\right) \in{\stackrel{\circ}{k_{i}}},(F, \mathbf{j}) \in S_{\mathcal{F}}$ and $0<t<1$ assume that $p_{\mathbf{x}_{i}}(t)=\mathbf{j}(i) \in \mathbf{Z}, i \in F$. Then $t=x_{i \mathbf{j}(i)}=x_{i^{\prime} \mathbf{j}\left(i^{\prime}\right)}$ for $i, i^{\prime} \in F$ and hence $\mathbf{x} \in E_{\mathcal{F}}$.

The composition of $P_{\mathcal{F}}^{<}$with the inclusion map $i: \vec{P}_{<}\left(X_{\mathcal{F}}\right)_{\mathbf{0}}^{\mathbf{k}+\mathbf{1}} \hookrightarrow \vec{P}\left(X_{\mathcal{F}}\right)_{\mathbf{0}}^{\mathbf{k}+\mathbf{1}}$ will be denoted by $\vec{P}_{\mathcal{F}}: D_{\mathcal{F}} \rightarrow \vec{P}\left(X_{\mathcal{F}}\right)_{\mathbf{0}}^{\mathbf{k}+\mathbf{1}}$.

Proposition 2.2. The map $\vec{P}_{\mathcal{F}}: D_{\mathcal{F}} \rightarrow \vec{P}\left(X_{\mathcal{F}}\right)_{\mathbf{0}}^{\mathbf{k}+\mathbf{1}}$ is a homotopy equivalence.

We prove Proposition 2.2 via the following two lemmas:

Lemma 2.3. The map $P_{\mathcal{F}}^{<}: D_{\mathcal{F}} \rightarrow \vec{P}_{<}\left(X_{\mathcal{F}}\right)_{\mathbf{0}}^{\mathbf{k}+\mathbf{1}}$ is a homotopy equivalence.

Proof. Define a reverse continuous map $Q: \vec{P}_{<}\left(\mathbf{R}^{n}\right)_{\mathbf{0}}^{\mathbf{k}+\mathbf{1}} \rightarrow \stackrel{\circ}{\mathbf{\Delta}}_{\mathbf{k}}$ as follows: For $\mathbf{p}=$ $\left(p_{1}, \ldots, p_{n}\right) \in \vec{P}_{<}\left(\mathbf{R}^{n}\right)_{\mathbf{0}}^{\mathbf{k}+\mathbf{1}}$ such that $p_{j}\left(x_{i j_{i}}\right)=j_{i}$ let $Q(\mathbf{p})=\left(x_{11}, \ldots, x_{1 k_{1}} ; \ldots ; x_{n 1}, \ldots, x_{n k_{n}}\right)$. Remark that $Q$ is well-defined and continuous since every $p_{i}$ is a homeomorphism; and that $Q$ cannot be extended to the space $\vec{P}\left(\mathbf{R}^{n}\right)_{\mathbf{0}}^{\mathbf{k}+\mathbf{1}}$ of non-decreasing directed paths. Remark moreover that $Q$ restricts to a map $Q_{\mathcal{F}}: \vec{P}_{<}\left(X_{\mathcal{F}}\right)_{\mathbf{0}}^{\mathbf{k}+\mathbf{1}} \rightarrow D_{\mathcal{F}}$.

It is obvious from the definitions that $Q \circ P$ is the identity map on $\AA_{\mathbf{k}}$ and hence that $Q_{\mathcal{F}} \circ P_{\mathcal{F}}$ is the identity map on $D_{\mathcal{F}}$. The map $P \circ Q: \vec{P}_{<}\left(\mathbf{R}^{n}\right)_{\mathbf{0}}^{\mathbf{k}+\mathbf{1}} \rightarrow \vec{P}_{<}\left(\mathbf{R}^{n}\right)_{\mathbf{0}}^{\mathbf{k}+\mathbf{1}}$ has the property: $((P \circ Q)(\mathbf{p}))_{i}\left(p_{i}^{-1}(j)\right)=j=p_{i}\left(p_{i}^{-1}(j)\right)$ and $((P \circ Q)(\mathbf{p}))_{i}(t) \notin \mathbf{Z}$ for $\mathbf{p} \in \vec{P}_{<}\left(\mathbf{R}^{n}\right)_{\mathbf{0}}^{\mathbf{k}+\mathbf{1}}$ and $t \notin Q_{i}(\mathbf{p}) \cup\{0,1\}$. That same property holds for all directed paths in the linear homotopy on $\vec{P}_{<}\left(\mathbf{R}^{n}\right)_{\mathbf{0}}^{\mathbf{k}+\mathbf{1}}$ given by $s \mapsto(1-s) \mathbf{p}+s(P \circ Q)(\mathbf{p}), 0 \leq s \leq 1$. Hence $P \circ Q$ restricts to a map $P_{\mathcal{F}} \circ Q_{\mathcal{F}}: \vec{P}_{<}\left(X_{\mathcal{F}}\right)_{\mathbf{0}}^{\mathbf{k}+\mathbf{1}} \rightarrow \vec{P}_{<}\left(X_{\mathcal{F}}\right)_{\mathbf{0}}^{\mathbf{k}+\mathbf{1}}$ that is homotopic to the identity map on $\vec{P}_{<}\left(X_{\mathcal{F}}\right)_{\mathbf{0}}^{\mathbf{k}+\mathbf{1}}$.

Lemma 2.4. The inclusion map $i: \vec{P}_{<}\left(X_{\mathcal{F}}\right)_{\mathbf{0}}^{\mathbf{k}+\mathbf{1}} \hookrightarrow \vec{P}\left(X_{\mathcal{F}}\right)_{\mathbf{0}}^{\mathbf{k}+\mathbf{1}}$ is a homotopy equivalence for every positive integer vector $\mathbf{k}$.

Proof. Let $\delta_{\mathbf{k}} \in \vec{P}_{<}\left(\mathbf{R}^{n}\right)_{\mathbf{0}}^{\mathbf{k}+\mathbf{1}}$ denote the linear path given by $\delta_{\mathbf{k}}(t)=t(\mathbf{k}+\mathbf{1})$. Then, for every $\mathbf{p} \in \vec{P}\left(\mathbf{R}^{n}\right)_{\mathbf{0}}^{\mathbf{k}+\mathbf{1}}$ and $0<s \leq 1$, the convex combination $\mathbf{p}_{s}:=(1-s) \mathbf{p}+s \delta_{\mathbf{k}}$ is strictly increasing and hence contained in $\vec{P}_{<}\left(\mathbf{R}^{n}\right)_{\mathbf{0}}^{\mathbf{k}+\mathbf{1}}$. For a given $\mathbf{p} \in \vec{P}_{<}\left(X_{\mathcal{F}}\right)_{\mathbf{0}}^{\mathbf{k}+\mathbf{1}}$, we want to choose $s>0$ small enough to ensure that $\mathbf{p}_{s}$ avoids $Y_{\mathcal{F}}$ (see $(1)$ ) and hence so that $\mathbf{p}_{s}$ is contained in $\vec{P}_{<}\left(X_{\mathcal{F}}\right)_{\mathbf{0}}^{\mathbf{k}+\mathbf{1}}$; and this in a way that makes the parameter $s$ depend continuously on the path $\mathbf{p}$.

Fix a norm and the associated metric $d$ on $\mathbf{R}^{n}$, e.g., the box norm. For every path $\mathbf{p} \in \vec{P}_{<}\left(X_{\mathcal{F}}\right)_{\mathbf{0}}^{\mathbf{k}+\mathbf{1}}$, the spaces $\mathbf{p}(I)$ and $Y_{\mathcal{F}} \cap[\mathbf{0}, \mathbf{k}+\mathbf{1}]$ are disjoint closed and hence compact 
subspaces of $[\mathbf{0}, \mathbf{k}+\mathbf{1}]$ with a positive distance $d(\mathbf{p}):=\max _{t \in I}\left(d\left(\mathbf{p}(t), Y_{\mathcal{F}}\right)\right.$ depending continuously on $\mathbf{p}$. Let $K:=\max _{1}^{n} k_{i}$ and let $s(\mathbf{p})=\frac{d(\mathbf{p})}{K}$. Then, for every $\mathbf{p} \in \vec{P}_{<}\left(X_{\mathcal{F}}\right)_{\mathbf{0}}^{\mathbf{k}+\mathbf{1}}$ one obtains: $d\left(\mathbf{p}, \mathbf{p}_{s}\right)=s(\mathbf{p})\left\|\mathbf{p}-\delta_{\mathbf{k}}\right\|<d(\mathbf{p})$ and in particular $d\left(\mathbf{p}_{s}, Y_{\mathcal{F}}\right)>0$.

Let $i: \vec{P}_{<}\left(X_{\mathcal{F}}\right)_{\mathbf{0}}^{\mathbf{k}+\mathbf{1}} \rightarrow \vec{P}\left(X_{\mathcal{F}}\right)_{\mathbf{0}}^{\mathbf{k}+\mathbf{1}}$ denote the inclusion map, and let $r: \vec{P}\left(X_{\mathcal{F}}\right)_{\mathbf{0}}^{\mathbf{k}+\mathbf{1}} \rightarrow$ $\vec{P}_{<}\left(X_{\mathcal{F}}\right)_{\mathbf{0}}^{\mathbf{k}+\mathbf{1}}$ denote the continuous map given by $r(\mathbf{p})=(1-s(\mathbf{p})) \mathbf{p}+s(\mathbf{p}) \delta_{\mathbf{k}}$. The continuous map $R: \vec{P}\left(X_{\mathcal{F}}\right)_{\mathbf{0}}^{\mathbf{k}+\mathbf{1}} \times I \rightarrow \vec{P}\left(X_{\mathcal{F}}\right)_{\mathbf{0}}^{\mathbf{k}+\mathbf{1}}$ given by $R(\mathbf{p}, t)=(1-t s(\mathbf{p})) \mathbf{p}+t s(\mathbf{p}) \delta_{\mathbf{k}}$ is a homotopy between the identity and $i \circ r$; its restriction to $\vec{P}_{<}\left(X_{\mathcal{F}}\right)_{\mathbf{0}}^{\mathbf{k}+\mathbf{1}}$ is a homotopy between the identity and $r \circ i$.

Remark: A variant of the proof above shows that spaces of increasing and of non-decreasing directed paths (as they arise in models for concurrency theory) are homotopy equivalent in a more general context.

\section{The Homology of $D_{\mathcal{F}}$}

In this section we state Theorem 3.1 that describes the homotopy type of the Alexander dual of $D_{\mathcal{F}}$ in the one-point compactification of $\stackrel{\circ}{\mathbf{k}}-$ a sphere of dimension $N=\sum_{i=1}^{n} k_{i}$. This result is then used to prove Theorem 1.3. Our main observation is the following homotopy decomposition of $\widehat{E_{\mathcal{F}}}$ (see $(3)$ and Definitions 1.2(i) and 2.1(iii)).

\section{Theorem 3.1.}

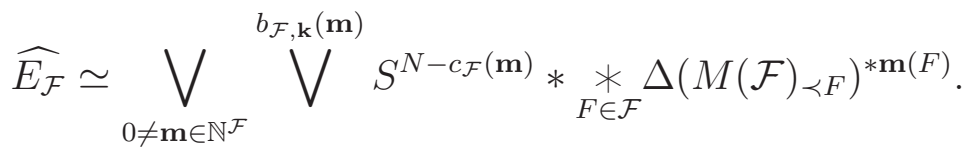

The proof of Theorem 3.1 is deferred to Section 4 .

Proof of Theorem 1.3: (i) If the integral homology $\tilde{H}_{*}\left(\Delta\left(M(\mathcal{F})_{\prec F}\right) ; \mathbb{Z}\right)$ is free for

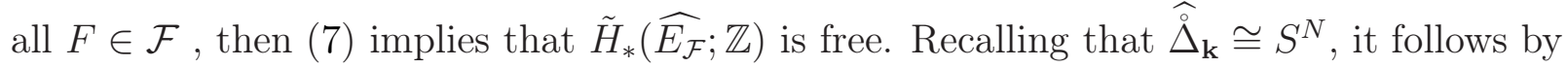
Alexander duality that for all $\ell$

$$
\begin{aligned}
& \tilde{H}_{\ell}\left(D_{\mathcal{F}} ; \mathbb{Z}\right)=\tilde{H}_{\ell}\left(\stackrel{\Delta}{\mathbf{k}}-E_{\mathcal{F}} ; \mathbb{Z}\right) \\
& =\tilde{H}_{\ell}\left(\widehat{\dot{\Delta}}_{\mathbf{k}}-\widehat{E_{\mathcal{F}}} ; \mathbb{Z}\right) \cong \tilde{H}_{N-\ell-1}\left(\widehat{E_{\mathcal{F}}} ; \mathbb{Z}\right) .
\end{aligned}
$$

Therefore $\tilde{H}_{\ell}\left(D_{\mathcal{F}} ; \mathbb{Z}\right)$ is free.

(ii) Recall that the behavior of the reduced Poincaré series $f_{\mathbb{K}}(\cdot)(\mathrm{cf} .(4))$; as a consequence, with respect to the wedge and join operations is given by

$$
\begin{aligned}
f_{\mathbb{K}}\left(Y_{1} \vee Y_{2}, t\right) & =f_{\mathbb{K}}\left(Y_{1}, t\right)+f_{\mathbb{K}}\left(Y_{2}, t\right) \\
f_{\mathbb{K}}\left(Y_{1} * Y_{2}, t\right) & =f_{\mathbb{K}}\left(Y_{1}, t\right) f_{\mathbb{K}}\left(Y_{2}, t\right) .
\end{aligned}
$$

Furthermore, if $Y$ is a subcomplex of $S^{N}$ then by Alexander duality

$$
f_{\mathbb{K}}\left(S^{N}-Y, t\right)=t^{N+1} f_{\mathbb{K}}\left(Y, t^{-1}\right) .
$$


Theorem 3.1 together with (8) imply that for any field $\mathbb{K}$

$$
f_{\mathbb{K}}\left(\widehat{E_{\mathcal{F}}}, t\right)=\sum_{0 \neq \mathbf{m} \in \mathbb{N}^{\mathcal{F}}} b_{\mathcal{F}, \mathbf{k}}(\mathbf{m}) t^{N-c_{\mathcal{F}}(\mathbf{m})+1} \prod_{F \in \mathcal{F}} f_{\mathbb{K}}\left(\Delta\left(M(\mathcal{F})_{\prec F}\right), t\right)^{\mathbf{m}(F)} .
$$

Combining Proposition 2.2 with (9) and (10) it follows that

$$
\begin{aligned}
f_{\mathbb{K}}\left(\vec{P}\left(X_{\mathcal{F}}\right)_{\mathbf{0}}^{\mathbf{k}+1}, t\right) & =f_{\mathbb{K}}\left(D_{\mathcal{F}}, t\right)=t^{N+1} f_{\mathbb{K}}\left(\widehat{E_{\mathcal{F}}}, t^{-1}\right) \\
& =\sum_{0 \neq \mathbf{m} \in \mathbb{N}^{\mathcal{F}}} b_{\mathcal{F}, \mathbf{k}}(\mathbf{m}) t^{\mathcal{F}_{\mathcal{F}}(\mathbf{m})} \prod_{F \in \mathcal{F}} f_{\mathbb{K}}\left(\Delta\left(M(\mathcal{F})_{\prec F}\right), t^{-1}\right)^{\mathbf{m}(F)} .
\end{aligned}
$$

\section{Homotopy Decomposition of $\widehat{E_{\mathcal{F}}}$}

In this Section we prove Theorem 3.1. Our basic approach is to apply the Wedge Lemma of Ziegler and Živaljević [18] to the cover $\left\{\Gamma_{(F, \mathbf{j})}:(F, \mathbf{j}) \in S_{\mathcal{F}}\right\}$ of $\widehat{E_{\mathcal{F}}}$. The actual proof depends on a number of preliminary results. For notations, we refer the reader to Definition 2.1 .

Definition 4.1. Let $R \subset S_{\mathcal{F}}$.

(i) Let

$$
G_{R}=\bigcap_{(F, \mathbf{j}) \in R} G_{(F, \mathbf{j})} \quad, \quad \Gamma_{R}=\bigcap_{(F, \mathbf{j}) \in R} \Gamma_{(F, \mathbf{j})}=G_{R} \cup\{\infty\} .
$$

(ii) $R$ is separated if $\mathbf{j}(i) \neq \mathbf{j}^{\prime}(i)$ for any $(F, \mathbf{j}) \neq\left(F^{\prime}, \mathbf{j}^{\prime}\right) \in R$ and $i \in F \cap F^{\prime}$.

For separated families $R \subset S_{\mathcal{F}}$ it is sometimes useful to represent $G_{R}$ by a diagram with $n$ rows such that the $i$-th row contains the coordinates $x_{i 1}<\cdots<x_{i k_{i}}$ of $\check{\Delta}_{k_{i}}$, and such that $x_{i j}, x_{i^{\prime} j^{\prime}}$ are connected by a dashed line iff $x_{i j}=x_{i^{\prime} j^{\prime}}$ for all $\mathbf{x} \in G_{R}$, i.e. iff there exists an $(F, \mathbf{j}) \in R$ such that $i, i^{\prime} \in F$ and $\mathbf{j}(i)=j$ and $\mathbf{j}\left(i^{\prime}\right)=j^{\prime}$.

Example 4.2. Let $k_{1}=k_{2}=k_{3}=2$ and let $R=\left\{\left(F_{i}, \mathbf{j}_{i}\right)\right\}_{i=1}^{3}$ where $F_{1}=\{1,2\}, F_{2}=$ $\{2,3\}, F_{3}=\{1,3\}$ and $\left(\mathbf{j}_{1}(1), \mathbf{j}_{1}(2)\right)=(1,1),\left(\mathbf{j}_{2}(2), \mathbf{j}_{2}(3)\right)=(2,1),\left(\mathbf{j}_{3}(1), \mathbf{j}_{3}(3)\right)=(2,2)$. The diagram of $G_{R}$ is depicted in Figure 2.

Definition 4.3. For $R \subset S_{\mathcal{F}}$ let $K_{R}$ be the directed graph on the vertex set $R$ with edges $(F, \mathbf{j}) \rightarrow\left(F^{\prime}, \mathbf{j}^{\prime}\right)$, where $(F, \mathbf{j})$ and $\left(F^{\prime}, \mathbf{j}^{\prime}\right)$ are distinct elements of $R$ that satisfy $F \cap F^{\prime} \neq \emptyset$ and $\mathbf{j}(i)<\mathbf{j}^{\prime}(i)$ for all $i \in F \cap F^{\prime}$. The family $R \subset S_{\mathcal{F}}$ is acyclic if $R$ is separated and if $K_{R}$ does not contain directed cycles. Let $A_{\mathcal{F}}$ denote the set of all acyclic subfamilies of $S_{\mathcal{F}}$.

The next two Propositions describe some properties of $\Gamma_{R}$ for separated families $R$. 


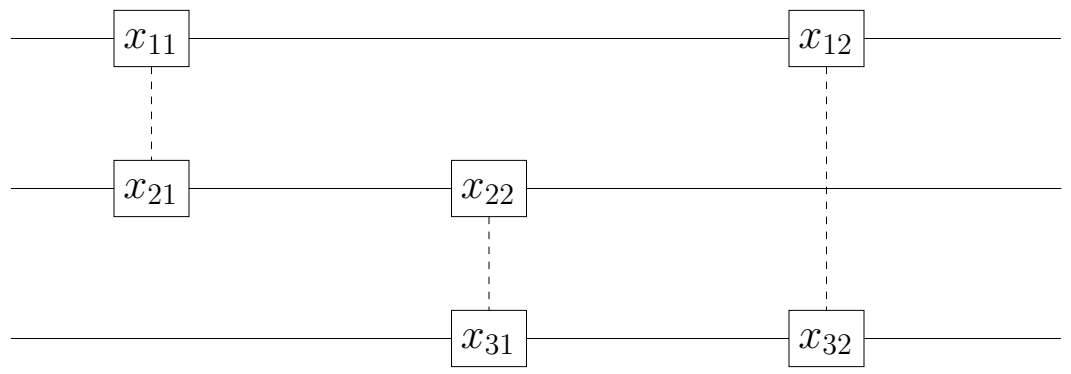

Figure 2: Diagram of $G_{R}$ (cf. Example 4.2)

Proposition 4.4. Let $R \subset S_{\mathcal{F}}$ be a separated family. Then:

(i) If $R \notin A_{\mathcal{F}}$ then $\Gamma_{R}=\{\infty\}$.

(ii) If $R \in A_{\mathcal{F}}$ then there is a homeomorphism

$$
\Gamma_{R} \cong S^{N-\sum_{(F, \mathbf{j}) \in R}(|F|-1)}
$$

Proof: (i) Let

$$
\left(F_{1}, \mathbf{j}_{1}\right) \rightarrow \cdots \rightarrow\left(F_{r}, \mathbf{j}_{r}\right) \rightarrow\left(F_{1}, \mathbf{j}_{1}\right)
$$

be a directed cycle in $K_{R}$. Then there exist

$$
i_{1} \in F_{1} \cap F_{2}, i_{2} \in F_{2} \cap F_{3}, \ldots, i_{r} \in F_{r} \cap F_{1}
$$

such that

$$
\mathbf{j}_{1}\left(i_{1}\right)<\mathbf{j}_{2}\left(i_{1}\right), \mathbf{j}_{2}\left(i_{2}\right)<\mathbf{j}_{3}\left(i_{2}\right), \cdots, \mathbf{j}_{r}\left(i_{r}\right)<\mathbf{j}_{1}\left(i_{r}\right) .
$$

We will show that $G_{R}=\emptyset$ and hence $\Gamma_{R}=\{\infty\}$. Indeed, suppose that $\left(\left(x_{i, 1}, \ldots, x_{i, k_{i}}\right)\right)_{i=1}^{n}$ is contained in $G_{R}$. We conclude from (12) - since $i_{j}, i_{j+1} \in F_{j+1}, j<r$, and $i_{1}, i_{r} \in F_{1}$ :

$$
\begin{aligned}
x_{i_{1}, \mathbf{j}_{1}\left(i_{1}\right)} & <x_{i_{1}, \mathbf{j}_{2}\left(i_{1}\right)}=x_{i_{2}, \mathbf{j}_{2}\left(i_{2}\right)}<x_{i_{2}, \mathbf{j}_{3}\left(i_{2}\right)}=x_{i_{3}, \mathbf{j}_{3}\left(i_{3}\right)}< \\
\cdots & <x_{i_{r-1}, \mathbf{j}_{r}\left(i_{r-1}\right)}=x_{i_{r}, \mathbf{j}_{r}\left(i_{r}\right)}<x_{i_{r}, \mathbf{j}_{1}\left(i_{r}\right)}=x_{i_{1}, \mathbf{j}_{1}\left(i_{1}\right)}
\end{aligned}
$$

a contradiction.

Example 4.5. Let $k_{1}=k_{2}=k_{3}=2$ and let $R=\left\{\left(F_{i}, \mathbf{j}_{i}\right)\right\}_{i=1}^{3}$ where $F_{1}=\{1,2\}, F_{2}=$ $\{2,3\}, F_{3}=\{1,3\}$ and $\left(\mathbf{j}_{1}(1), \mathbf{j}_{1}(2)\right)=(2,1),\left(\mathbf{j}_{2}(2), \mathbf{j}_{2}(3)\right)=(2,1),\left(\mathbf{j}_{3}(1), \mathbf{j}_{3}(3)\right)=(1,2)$. Then $\left(F_{1}, \mathbf{j}_{1}\right) \rightarrow\left(F_{2}, \mathbf{j}_{2}\right) \rightarrow\left(F_{3}, \mathbf{j}_{3}\right) \rightarrow\left(F_{1}, \mathbf{j}_{1}\right)$ is a cycle in $K_{R}$ and thus $G_{R}=\emptyset$, as is also clear from the diagram of $G_{R}$ in Figure 3.

(ii) For $(F, \mathbf{j}) \in S_{\mathcal{F}}$ define

$$
V_{(F, \mathbf{j})}=\left\{\left(x_{i 1}, \ldots, x_{i k_{i}}\right)_{i=1}^{n} \in \prod_{i=1}^{n} \mathbb{R}^{k_{i}}: x_{i \mathbf{j}(i)}=x_{i^{\prime} \mathbf{j}\left(i^{\prime}\right)} \text { for all } i, i^{\prime} \in F\right\} .
$$




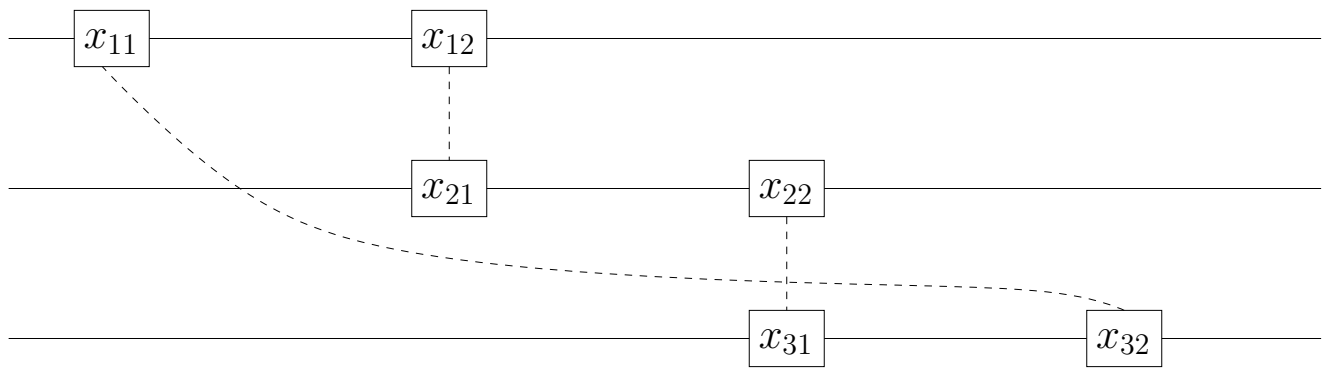

Figure 3: If $K_{R}$ has directed cycles then $G_{R}=\emptyset$ (cf. Example 4.5)

For $R \subset S_{\mathcal{F}}$, let $V_{R}=\bigcap_{(F, \mathbf{j}) \in R} V_{(F, \mathbf{j})} \subset \mathbb{R}^{N}$. If the family $R$ is separated, then $V_{R}$ is a linear subspace of codimension $\sum_{(F, \mathbf{j}) \in R}(|F|-1)$. If $R$ is acyclic, one can easily find an element $\mathbf{x} \in V_{R} \cap \stackrel{\circ}{\Delta}_{\mathbf{k}}$

For such a chosen solution $\mathbf{x}$ and for $\mathbf{v} \in S\left(V_{R}\right)=\left\{\mathbf{u} \in V_{R}:\|\mathbf{u}\|=1\right\} \subset D\left(V_{R}\right)=\{\mathbf{u} \in$ $\left.V_{R}:\|\mathbf{u}\| \leq 1\right\}$ let $\alpha(\mathbf{v})=\min \left\{t>0 \mid \mathbf{x}+t \mathbf{v} \in \partial \Delta_{\mathbf{k}}\right\}$; hence, for $\mathbf{w} \in \partial \Delta_{\mathbf{k}}$, one has that $\alpha\left(\frac{\mathbf{w}-\mathbf{x}}{\|\mathbf{w}-\mathbf{x}\|}\right)=\|\mathbf{w}-\mathbf{x}\|$. This recipe defines a continuous map $\alpha$ from $S\left(V_{R}\right)$ to the positive reals since $\alpha(\mathbf{v})$ is locally obtained as the minimum among the solutions to a number of linear equations.

We define a (scaling) map

$$
\Phi_{R}: \Gamma_{R}=V_{R} \cap \Delta_{\mathbf{k}} / V_{R} \cap \partial \Delta_{\mathbf{k}} \rightarrow D\left(V_{R}\right) /_{S\left(V_{R}\right)} \cong S^{N-\sum_{(F, \mathbf{j}) \in R}(|F|-1)}
$$

by

$$
\Phi_{R}(\mathbf{w})= \begin{cases}\frac{1}{\alpha\left(\frac{\mathbf{w}-\mathbf{x}}{\|\mathbf{w}-\mathbf{x}\|}\right)}(\mathbf{w}-\mathbf{x}) & \mathbf{w} \neq \mathbf{x} \\ \mathbf{0} & \mathbf{w}=\mathbf{x} .\end{cases}
$$

The map $\Phi_{R}$ is indeed a homeomorphism with inverse $\Psi_{R}: D\left(V_{R}\right) / S\left(V_{R}\right) \rightarrow \Gamma_{R}$ given by

$$
\Psi_{R}(\mathbf{v})= \begin{cases}\mathbf{x}+\alpha\left(\frac{\mathbf{v}}{\|\mathbf{v}\|}\right) \mathbf{v} & \mathbf{v} \neq \mathbf{0} \\ \mathbf{x} & \mathbf{v}=\mathbf{0}\end{cases}
$$

Proposition 4.6. Let $R, R^{\prime} \in A_{\mathcal{F}}$. Then the following two conditions are equivalent:

(a) $\Gamma_{R} \subset \Gamma_{R^{\prime}}$.

(b) For any $\left(F^{\prime}, \mathbf{j}^{\prime}\right) \in R^{\prime}$ there exists an $(F, \mathbf{j}) \in R$ such that $F^{\prime} \subset F$ and $\mathbf{j}^{\prime}=(\mathbf{j})_{\mid F^{\prime}}$.

Proof: Clearly (b) implies (a). To show the other direction, assume that $\Gamma_{R} \subset \Gamma_{R^{\prime}}$ and let $\left(F^{\prime}, \mathbf{j}^{\prime}\right) \in R^{\prime}$. Let $R_{1}=R \cup\left\{\left(F^{\prime}, \mathbf{j}^{\prime}\right)\right\}$, then

$$
\Gamma_{R}=\Gamma_{R} \cap \Gamma_{R^{\prime}} \subset \Gamma_{R_{1}} \subset \Gamma_{R}
$$


hence $\Gamma_{R_{1}}=\Gamma_{R} \neq\{\infty\}$. It follows that if $R_{1}$ is separated then it must be acyclic. But this would imply, using Proposition 4.4(ii), that

$$
\operatorname{dim} \Gamma_{R_{1}}=\operatorname{dim} \Gamma_{R}-\left(\left|F^{\prime}\right|-1\right)<\operatorname{dim} \Gamma_{R},
$$

in contradiction with $\Gamma_{R_{1}}=\Gamma_{R}$. Hence $R_{1}$ is not separated and therefore

$$
S=\left\{(F, \mathbf{j}) \in R: F \cap F^{\prime} \neq \emptyset \quad \& \quad(\mathbf{j})_{\mid F \cap F^{\prime}}=\left(\mathbf{j}^{\prime}\right)_{\mid F \cap F^{\prime}}\right\} \neq \emptyset .
$$

We claim that $|S|=1$. Otherwise there exist $\left(F_{1}, \mathbf{j}_{1}\right) \neq\left(F_{2}, \mathbf{j}_{2}\right) \in R$ and $i_{1} \in F_{1} \cap F^{\prime}, i_{2} \in$ $F_{2} \cap F^{\prime}$ such that $\mathbf{j}_{1}\left(i_{1}\right)=\mathbf{j}^{\prime}\left(i_{1}\right)$ and $\mathbf{j}_{2}\left(i_{2}\right)=\mathbf{j}^{\prime}\left(i_{2}\right)$. It follows that if $\mathbf{x}=\left(x_{i 1}, \ldots, x_{i k_{i}}\right)_{i=1}^{n} \in$ $G_{R_{1}}$ then for all $i_{1}^{\prime} \in F_{1}, i_{2}^{\prime} \in F_{2}$

$$
\begin{aligned}
x_{i_{1}^{\prime} \mathbf{j}_{1}\left(i_{1}^{\prime}\right)} & =x_{i_{1} \mathbf{j}_{1}\left(i_{1}\right)}=x_{i_{1} \mathbf{j}^{\prime}\left(i_{1}\right)} \\
& =x_{i_{2} \mathbf{j}^{\prime}\left(i_{2}\right)}=x_{i_{2} \mathbf{j}_{2}\left(i_{2}\right)}=x_{i_{2}^{\prime} \mathbf{j}_{2}\left(i_{2}^{\prime}\right)} .
\end{aligned}
$$

Since $R$ is separated, (13) implies that $F_{1} \cap F_{2}=\emptyset$. Let $F_{3}=F_{1} \cup F_{2}$ and let $\mathbf{j}_{3} \in\left[\mathbf{k}_{F_{3}}\right]$ be given by

Writing

$$
\mathbf{j}_{3}(i)= \begin{cases}\mathbf{j}_{1}(i) & i \in F_{1} \\ \mathbf{j}_{2}(i) & i \in F_{2}\end{cases}
$$

$$
R_{2}=R \backslash\left\{\left(F_{1}, \mathbf{j}_{1}\right),\left(F_{2}, \mathbf{j}_{2}\right)\right\} \cup\left\{\left(F_{3}, \mathbf{j}_{3}\right)\right\},
$$

it follows from (13) that $\Gamma_{R}=\Gamma_{R_{1}} \subset \Gamma_{R_{2}} \subset \Gamma_{R}$. Therefore $\Gamma_{R_{2}}=\Gamma_{R} \neq\{\infty\}$. As $R_{2}$ is separated, it follows that $R_{2} \in A_{\mathcal{F}}$ and hence by Proposition 4.4(ii):

$$
\operatorname{dim} \Gamma_{R_{2}}=\operatorname{dim} \Gamma_{R}+\left(\left|F_{1}\right|-1\right)+\left(\left|F_{2}\right|-1\right)-\left(\left|F_{1} \cup F_{2}\right|-1\right)=\operatorname{dim} \Gamma_{R}-1,
$$

in contradiction with $\Gamma_{R_{2}}=\Gamma_{R}$. Therefore $|S|=1$.

Write $S=\left\{\left(F_{1}, \mathbf{j}_{1}\right)\right\}$ and let $i_{1} \in F_{1} \cap F^{\prime}$. Then $\mathbf{j}_{1}\left(i_{1}\right)=\mathbf{j}^{\prime}\left(i_{1}\right)$. It follows that if $\mathbf{x}=\left(x_{i 1}, \ldots, x_{i k_{i}}\right)_{i=1}^{n} \in G_{R_{1}}$ then for all $i_{1}^{\prime} \in F_{1}, i^{\prime} \in F^{\prime}$

$$
x_{i_{1}^{\prime} \mathbf{j}_{1}\left(i_{1}^{\prime}\right)}=x_{i_{1} \mathbf{j}_{1}\left(i_{1}\right)}=x_{i_{1} \mathbf{j}^{\prime}\left(i_{1}\right)}=x_{i^{\prime} \mathbf{j}^{\prime}\left(i^{\prime}\right)} .
$$

Let $F_{4}=F_{1} \cup F^{\prime}$ and let $\mathbf{j}_{4} \in\left[\mathbf{k}_{F_{4}}\right]$ be given by

$$
\mathbf{j}_{4}(i)= \begin{cases}\mathbf{j}_{1}(i) & i \in F_{1}, \\ \mathbf{j}^{\prime}(i) & i \in F^{\prime}\end{cases}
$$

Note that $\mathbf{j}_{4}$ is well defined by (14). Writing

$$
R_{3}=R \backslash\left\{\left(F_{1}, \mathbf{j}_{1}\right)\right\} \cup\left\{\left(F_{4}, \mathbf{j}_{4}\right)\right\},
$$

it follows from (14) that

$$
\Gamma_{R}=\Gamma_{R_{1}} \subset \Gamma_{R_{3}} \subset \Gamma_{R},
$$

hence $\Gamma_{R_{3}}=\Gamma_{R} \neq\{\infty\}$. Furthermore, $|S|=1$ implies that $R_{3}$ is separated. Therefore by Proposition 4.4(ii):

$$
\operatorname{dim} \Gamma_{R_{3}}=\operatorname{dim} \Gamma_{R}+\left(\left|F_{1}\right|-1\right)-\left(\left|F_{4}\right|-1\right) .
$$

It follows that $\left|F_{1}\right|=\left|F_{4}\right|=\left|F_{1} \cup F^{\prime}\right|$. Hence $F^{\prime} \subset F_{1}$ and $\mathbf{j}^{\prime}=\left(\mathbf{j}_{1}\right)_{\mid F^{\prime}}$. 
Let $Q$ be the intersection poset of the cover $\left\{\Gamma_{(F, \mathbf{j})}:(F, \mathbf{j}) \in S_{\mathcal{F}}\right\}$ of $\widehat{E_{\mathcal{F}}}$ ordered by reverse inclusion: An element $q$ of $Q$ corresponds to an intersection $U_{q}$ of sets in the cover, i.e. $U_{q}=\Gamma_{R}$ for $R \subset S_{\mathcal{F}}$, and $q^{\prime} \leq q$ in $Q$ iff $U_{q} \subset U_{q^{\prime}} . Q$ has a maximal element $\hat{1}$ that corresponds to $U_{\widehat{1}}=\{\infty\}$. Fix a $\widehat{1} \neq q \in Q$ and let $R \subset S_{\mathcal{F}}$ be a family of minimal cardinality such that $U_{q}=\Gamma_{R}$. The assumption that $\mathcal{F}$ is upward closed implies that $R$ is a separated family. Indeed, suppose $u^{\prime}=\left(F^{\prime}, \mathbf{j}^{\prime}\right) \neq u^{\prime \prime}=\left(F^{\prime \prime}, \mathbf{j}^{\prime \prime}\right) \in R$ and there exists some $i_{0} \in F^{\prime} \cap F^{\prime \prime}$ such that $\mathbf{j}^{\prime}\left(i_{0}\right)=\mathbf{j}^{\prime \prime}\left(i_{0}\right)$. Let $\infty \neq \mathbf{x}=\left(x_{i 1}, \ldots, x_{i k_{i}}\right)_{i=1}^{n} \in \Gamma_{R}$. Then for any $i \in F^{\prime} \cap F^{\prime \prime}$

$$
x_{i \mathrm{j}^{\prime}(i)}=x_{i_{0} \mathbf{j}^{\prime}\left(i_{0}\right)}=x_{i_{0} \mathbf{j}^{\prime \prime}\left(i_{0}\right)}=x_{i \mathbf{j}^{\prime \prime}(i)},
$$

hence $\mathbf{j}^{\prime}(i)=\mathbf{j}^{\prime \prime}(i)$. Let $u=(F, \mathbf{j}) \in S_{\mathcal{F}}$ where $F=F^{\prime} \cup F^{\prime \prime}$ and

$$
\mathbf{j}(i)= \begin{cases}\mathbf{j}^{\prime}(i) & i \in F^{\prime}, \\ \mathbf{j}^{\prime \prime}(i) & i \in F^{\prime \prime} .\end{cases}
$$

Then $\Gamma_{R}=\Gamma_{R-\left\{u^{\prime}, u^{\prime \prime}\right\} \cup\{u\}}$, contradicting the minimality of $R$. Thus $R$ is separated. By Proposition 4.4(i), the assumption $q \neq \widehat{1}$ implies that $R \in A_{\mathcal{F}}$; cf. Definition 4.3.

We next study the topology of the order complex $\Delta\left(Q_{<q}\right)$.

Proposition 4.7. Fix $\widehat{1} \neq q \in Q$ and write $U_{q}=\Gamma_{R}$ where $R=\left\{\left(F_{\ell}, \mathbf{j}_{\ell}\right)\right\}_{\ell=1}^{r} \in A_{\mathcal{F}}$. Then there is a homeomorphism

$$
\Delta\left(Q_{<q}\right) \cong \Delta\left(M(\mathcal{F})_{\prec F_{1}}\right) * \cdots * \Delta\left(M(\mathcal{F})_{\prec F_{r}}\right) * S^{r-2} .
$$

Proof: Let $M(\mathcal{F})_{\leq F_{\ell}}^{*}$ denote the poset obtained by appending to $M(\mathcal{F})_{\preceq F_{\ell}}$ a minimal element $0_{\ell}$. Denote

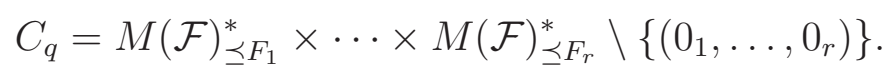

Define a mapping $\gamma: C_{q} \rightarrow A_{\mathcal{F}}$ as follows. Let $\alpha=\left(\alpha_{1}, \ldots, \alpha_{r}\right) \in C_{q}$ and let

$$
L(\alpha)=\left\{1 \leq \ell \leq r: \alpha_{\ell} \neq 0_{\ell}\right\} .
$$

Note that $L(\alpha) \neq \emptyset$. For $\ell \in L(\alpha)$ write $\alpha_{\ell}=\mathcal{G}_{\ell} \in M(\mathcal{F})$ and let

$$
\gamma(\alpha)=\bigcup_{\ell \in L(\alpha)}\left\{\left(F,\left(\mathbf{j}_{\ell}\right)_{\mid F}\right): F \in \mathcal{G}_{\ell}\right\} .
$$

Define an order preserving map $\theta: C_{q} \rightarrow Q_{\leq q}$ as follows: For $\alpha \in C_{q}$ let $\theta(\alpha)$ be the element of $Q$ that satisfies $U_{\theta(\alpha)}=\Gamma_{\gamma(\alpha)}$.

Lemma 4.8. $\theta$ is a poset isomorphism. 
Proof: To show surjectivity, let $q^{\prime} \leq q$ with $U_{q^{\prime}}=\Gamma_{R^{\prime}}$ for some $R^{\prime} \in A_{\mathcal{F}}$. Then $\Gamma_{R}=$ $U_{q} \subset U_{q^{\prime}}=\Gamma_{R^{\prime}}$ and hence, by Proposition 4.6, there exists an $\alpha \in C_{q}$ such that $\gamma(\alpha)=R^{\prime}$. Therefore $U_{\theta(\alpha)}=\Gamma_{\gamma(\alpha)}=\Gamma_{R^{\prime}}=U_{q^{\prime}}$ and so $\theta(\alpha)=q^{\prime}$. To show injectivity, assume that $\theta(\alpha)=\theta\left(\alpha^{\prime}\right)$ for some $\alpha, \alpha^{\prime} \in C_{q}$. Then $\Gamma_{\gamma(\alpha)}=U_{\theta(\alpha)}=U_{\theta\left(\alpha^{\prime}\right)}=\Gamma_{\gamma\left(\alpha^{\prime}\right)}$ and therefore $\gamma(\alpha)=\gamma\left(\alpha^{\prime}\right)$ by Proposition 4.6. As $\gamma$ is clearly injective, it follows that $\alpha=\alpha^{\prime}$.

Recall the following result of Walker (Theorem 5.1(d) in [17]).

Theorem 4.9 (Walker [17]). For $1 \leq i \leq r$ let $T_{i}$ be a finite poset with minimal element $0_{i}$ and maximal element $1_{i}$. Let $T=T_{1} \times \cdots \times T_{r}$ and $\mathbf{0}=\left(0_{1}, \ldots, 0_{r}\right), \mathbf{1}=\left(1_{1}, \ldots, 1_{r}\right) \in T$. Let $\widehat{T}_{i}=T_{i}-\left\{\left(0_{i}, 1_{i}\right)\right\}$ and $\widehat{T}=T-\{\mathbf{0}, \mathbf{1}\}$. Then there is a homeomorphism

$$
\Delta(\widehat{T}) \cong \Delta\left(\widehat{T}_{1}\right) * \cdots * \Delta\left(\widehat{T}_{r}\right) * S^{r-2} .
$$

For $1 \leq i \leq r$ let $T_{i}=M(\mathcal{F})_{\left\llcorner F_{i}\right.}^{*}$. Then $\widehat{T}_{i}=M(\mathcal{F})_{\prec F_{i}}$ and $\widehat{T}=C_{q}-\left\{\left(\left\{F_{1}\right\}, \ldots,\left\{F_{r}\right\}\right)\right\}$. Lemma 4.8 thus implies that $\widehat{T} \cong Q_{<q}$. Therefore by Theorem 4.9:

$$
\begin{aligned}
\Delta\left(Q_{<q}\right) \cong \Delta(\widehat{T}) \cong \Delta\left(\widehat{T}_{1}\right) * \cdots * \Delta\left(\widehat{T}_{r}\right) * S^{r-2} \\
\\
=\Delta\left(M(\mathcal{F})_{\prec F_{1}}\right) * \cdots * \Delta\left(M(\mathcal{F})_{\prec F_{r}}\right) * S^{r-2} .
\end{aligned}
$$

Definition 4.10. For a function $0 \neq \mathbf{m} \in \mathbb{N}^{\mathcal{F}}$, let $A_{\mathcal{F}}(\mathbf{m})$ denote the set of all $R \in A_{\mathcal{F}}$ such that $\left|\left\{\mathbf{j} \in\left[\mathbf{k}_{F}\right] \mid(F, \mathbf{j}) \in R\right\}\right|=\mathbf{m}(F)$ for all $F \in \mathcal{F}$.

The final ingredient needed for the proof of Theorem 3.1 is the following computation (see (3) and Definition 1.2(i)).

Proposition 4.11. Let $0 \neq \mathbf{m} \in \mathbb{N}^{\mathcal{F}}$. Then:

$$
\left|A_{\mathcal{F}}(\mathbf{m})\right|=b_{\mathcal{F}, \mathbf{k}}(\mathbf{m})=\frac{a\left(T_{\mathcal{F}}(\mathbf{m})\right)}{\prod_{F \in \mathcal{F}} \mathbf{m}(F) !} \prod_{j=1}^{n}\left(\begin{array}{c}
k_{j} \\
\sum_{F \ni j} \mathbf{m}(F)
\end{array}\right) .
$$

Proof: Let $\tilde{\mathfrak{A}}\left(T_{\mathcal{F}}(\mathbf{m})\right)$ denote the set of all acyclic orientations of $T_{\mathcal{F}}(\mathbf{m})$ such that $(F, i) \rightarrow$ $\left(F, i^{\prime}\right)$ for all $F \in \mathcal{F}$ and $1 \leq i<i^{\prime} \leq \mathbf{m}(F)$. Then

$$
\left|\tilde{\mathfrak{A}}\left(T_{\mathcal{F}}(\mathbf{m})\right)\right|=\frac{a\left(T_{\mathcal{F}}(\mathbf{m})\right)}{\prod_{F \in \mathcal{F}} \mathbf{m}(F) !} .
$$

Define a mapping

$$
\tau: A_{\mathcal{F}}(\mathbf{m}) \rightarrow \tilde{\mathfrak{A}}\left(T_{\mathcal{F}}(\mathbf{m})\right) \times \prod_{i=1}^{n}\left(\begin{array}{c}
{\left[k_{i}\right]} \\
\sum_{F \ni i} \mathbf{m}(F)
\end{array}\right)
$$


as follows. Let $R \in A_{\mathcal{F}}(\mathbf{m})$. For $1 \leq i \leq n$ let

$$
B_{i}=\{\mathbf{j}(i):(F, \mathbf{j}) \in R \text { and } i \in F\} \in\left(\begin{array}{c}
{\left[k_{i}\right]} \\
\sum_{F \ni i} \mathbf{m}(F)
\end{array}\right) .
$$

Write

$$
R=\bigcup_{\{F \in \mathcal{F}: \mathbf{m}(F)>0\}}\left\{\left(F, \mathbf{j}_{F, \ell}\right): 1 \leq \ell \leq \mathbf{m}(F)\right\}
$$

where $\mathbf{j}_{F, \ell} \in \mathbf{k}_{F}$ for all $1 \leq \ell \leq \mathbf{m}(F)$ and

$$
\mathbf{j}_{F, 1}(i)<\cdots<\mathbf{j}_{F, \mathbf{m}(F)}(i)
$$

for all $i \in F$. Define an orientation $\alpha \in \tilde{\mathfrak{A}}\left(T_{\mathcal{F}}(\mathbf{m})\right)$ as follows. Let $e=\left\{(F, s),\left(F^{\prime}, s^{\prime}\right)\right\}$ be an edge of $T_{\mathcal{F}}(\mathbf{m})$. Define $\alpha(e)=\left((F, s),\left(F^{\prime}, s^{\prime}\right)\right)$ if either $F=F^{\prime}$ and $s<s^{\prime}$, or if $F \neq F^{\prime}$ and $\mathbf{j}_{F, s}(i)<\mathbf{j}_{F^{\prime}, s^{\prime}}(i)$ for some (and therefore all) $i \in F \cap F^{\prime}$. Now let

$$
\tau(R)=\left(\alpha, B_{1}, \ldots, B_{n}\right) .
$$

It is straightforward to check that $\tau$ is bijective. This proves Proposition 4.11.

Example 4.12. To illustrate the bijection $\tau$ from the proof of Claim 4.11 consider the family $\mathcal{F}=\{F \subset[4]:|F| \geq 2\}$ and let $n=4,\left(k_{1}, k_{2}, k_{3}, k_{4}\right)=(4,5,4,2)$. Let $F_{1}=\{1,2\}$, $F_{2}=\{2,3\}$ and $F_{3}=\{1,3,4\}$ and for $F \in \mathcal{F}$ let

$$
\mathbf{m}(F)= \begin{cases}2 & F=F_{1} \text { or } F=F_{2}, \\ 1 & F=F_{3}, \\ 0 & \text { otherwise }\end{cases}
$$

Let $R \in A_{\mathcal{F}}(\mathbf{m})$ satisfy $\tau(R)=\left(\alpha, B_{1}, B_{2}, B_{3}, B_{4}\right)$ where

$$
\left(B_{1}, B_{2}, B_{3}, B_{4}\right)=(\{2,3,4\},\{1,2,4,5\},\{1,3,4\},\{2\})
$$

and the orientation $\alpha$ on the (complete) graph $T_{\mathcal{F}}(\mathbf{m})$ is given by the total order

$$
\left(F_{2}, 1\right) \rightarrow\left(F_{1}, 1\right) \rightarrow\left(F_{1}, 2\right) \rightarrow\left(F_{3}, 1\right) \rightarrow\left(F_{2}, 2\right) .
$$

The reconstruction of $R$ from $\tau(R)$ is depicted in Figure 4.

Proof of Theorem 3.1: Consider the cover $\left\{\Gamma_{(F, \mathbf{j})}:(F, \mathbf{j}) \in S_{\mathcal{F}}\right\}$ of $\widehat{E_{\mathcal{F}}}$, and its associated intersection poset $Q$ as above. By Proposition 4.4(ii), if $\widehat{1} \neq q \in Q$ then $U_{q}$ is a sphere pointed at $\infty$. Furthermore, if $q<p \in Q$ then the injection $U_{p} \rightarrow U_{q}$ is a pointed embedding of a sphere (or the point $\infty$ if $p=\widehat{1}$ ) into a higher dimensional sphere and 


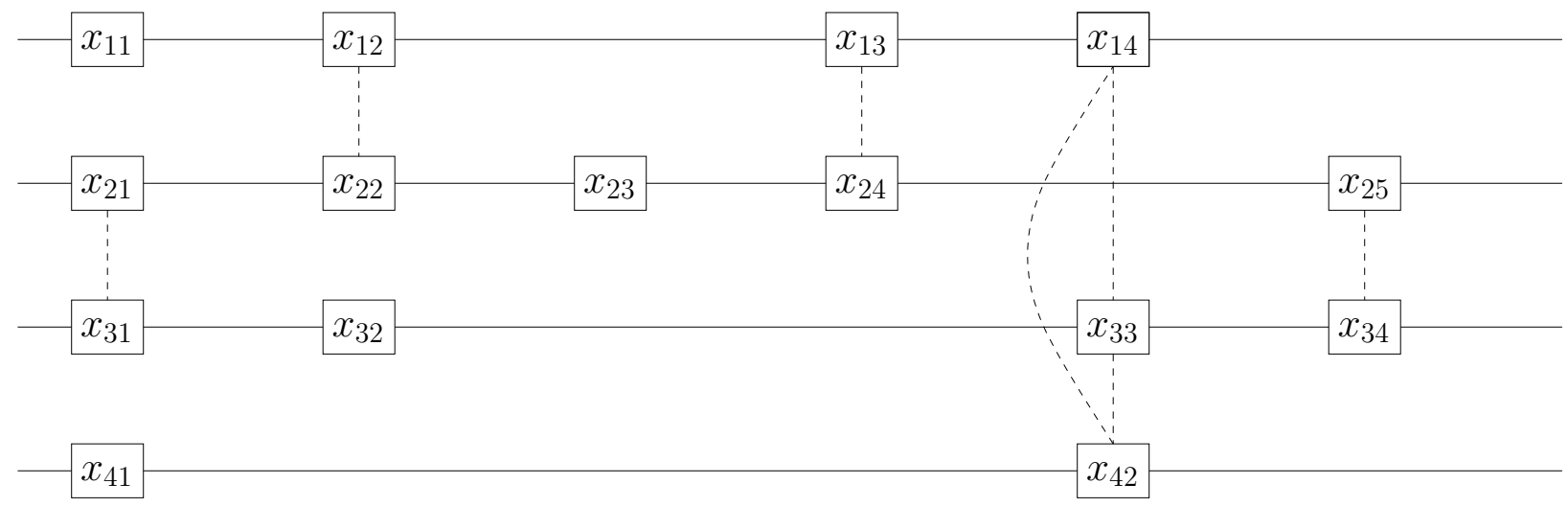

Figure 4: Reconstruction of $R$ from $\tau(R)$ (cf. Example 4.12)

is thus homotopic to the constant map $U_{p} \rightarrow \infty \in U_{q}$. Therefore by the Wedge Lemma (Lemma 1.8 in [18]) there is a homotopy equivalence

$$
\widehat{E_{\mathcal{F}}} \simeq \bigvee_{q \in Q} \Delta\left(Q_{<q}\right) * U_{q}
$$

We next determine the contribution of each $q \in Q$ to (17). If $q=\hat{1}$ then $\Delta\left(Q_{<q}\right) * U_{q}$ is contractible to the point $\infty$ and hence does not contribute to (17). Suppose $q<\widehat{1}$ and let $U_{q}=\Gamma_{R}$ where $R=\left\{\left(F_{\ell}, \mathbf{j}_{\ell}\right)\right\}_{\ell=1}^{r} \in A_{\mathcal{F}}$. Combining Proposition 4.4(ii) and (15) it follows that

$$
\begin{aligned}
\Delta\left(Q_{<q}\right) * U_{q} & \cong \Delta\left(M(\mathcal{F})_{\prec F_{1}}\right) * \cdots * \Delta\left(M(\mathcal{F})_{\prec F_{r}}\right) * S^{r-2} * S^{N-\sum_{(F, \mathbf{j}) \in R}(|F|-1)} \\
& \cong S^{N-\sum_{i=1}^{r}\left(\left|F_{i}\right|-2\right)-1} * \underset{i=1}{*} \Delta\left(M(\mathcal{F})_{\prec F_{i}}\right) .
\end{aligned}
$$

Therefore, if $R \in A_{\mathcal{F}}(\mathbf{m})$ and $U_{q}=\Gamma_{R}$ then (cf. (3))

$$
\Delta\left(Q_{<q}\right) * U_{q} \cong S^{N-c_{\mathcal{F}}(\mathbf{m})} * \underset{F \in \mathcal{F}}{*} \Delta\left(M(\mathcal{F})_{\prec F}\right)^{* \mathbf{m}(F)} .
$$

Theorem 3.1 now follows from (17), (18) and Proposition 4.11.

\section{Applications}

In this section we use Theorem 1.3 to study several specific Euclidean pattern spaces. 


\subsection{The Homology of $\vec{P}\left(\mathbb{R}^{n} \backslash \mathbb{Z}^{n}\right)_{0}^{\mathbf{k}+1}$}

As noted earlier, $\mathbb{R}^{n} \backslash \mathbb{Z}^{n}=X_{\mathcal{F}}$ where $\mathcal{F}$ consists of the single set $[n]$. Since $\Delta\left(M(\mathcal{F})_{\prec[n]}\right)$ is the empty complex $\{\emptyset\}$ it follows that $f_{\mathbb{K}}\left(\Delta\left(M(\mathcal{F})_{\prec[n]}\right), t\right)=1$. If $\mathbf{m}([n])=m>0$ then $b_{\mathcal{F}, \mathbf{k}}(\mathbf{m})=\prod_{i=1}^{n}\left(\begin{array}{c}k_{i} \\ m\end{array}\right)$ and $c_{\mathcal{F}}(\mathbf{m})=m(n-2)+1$. Theorem 1.3 implies that

$$
f_{\mathbb{K}}\left(\vec{P}\left(\mathbb{R}^{n} \backslash \mathbb{Z}^{n}\right)_{\mathbf{0}}^{\mathbf{k}+\mathbf{1}}, t\right)=\sum_{m \geq 1} \prod_{i=1}^{n}\left(\begin{array}{l}
k_{i} \\
m
\end{array}\right) t^{m(n-2)+1} .
$$

Since $\tilde{H}_{*}\left(\Delta\left(M(\mathcal{F})_{\prec[n]}\right)\right)=\tilde{H}_{-1}(\{\emptyset\})=\mathbb{Z}$ is free, it follows that $\tilde{H}_{\ell}\left(\vec{P}\left(\mathbb{R}^{n} \backslash \mathbb{Z}^{n}\right)_{\mathbf{0}}^{\mathbf{k}+\mathbf{1}}\right)$ is free of rank $\prod_{i=1}^{n}\left(\begin{array}{l}k_{i} \\ m\end{array}\right)$ if $\ell=(n-2) m>0$, and is zero otherwise. This recovers the above mentioned Theorem 1.1 of Raussen and Ziemiański [14].

\subsection{Binary Path Spaces}

The binary path space associated with an upward closed $\mathcal{F} \subset 2^{[n]}$ is $\vec{P}\left(X_{\mathcal{F}}\right)_{\mathbf{0}}^{\mathbf{2}}$ where $\mathbf{2}=$ $(2, \ldots, 2)$. Note that $\vec{P}\left(X_{\mathcal{F}}\right)_{\mathbf{0}}^{\mathbf{2}}$ is homotopy equivalent to the diagonal subspace arrangement

$$
\mathbb{R}^{n}-\bigcup_{F=\left\{i_{1}, \ldots, i_{\ell}\right\} \in \mathcal{F}}\left\{x=\left(x_{1}, \ldots, x_{n}\right) \in \mathbb{R}^{n}: x_{i_{1}}=\cdots=x_{i_{\ell}}\right\} .
$$

The general formula (5) for the Poincaré series of $\vec{P}\left(X_{\mathcal{F}}\right)_{\mathbf{0}}^{\mathbf{k}+\mathbf{1}}$ simplifies in this case as follows. Let $\mathbf{k}=\mathbf{1}$ and let $0 \neq \mathbf{m} \in \mathbb{N}^{\mathcal{F}}$. Then $b_{\mathcal{F}, \mathbf{1}}(\mathbf{m})=1$ if both $\mathbf{m}(F) \leq 1$ for all $F \in \mathcal{F}$, and $\{F: \mathbf{m}(F)=1\} \in M(\mathcal{F})$. Otherwise $b_{\mathcal{F}, \mathbf{1}}(\mathbf{m})=0$. Hence, by $(5)$

$$
f_{\mathbb{K}}\left(\vec{P}\left(X_{\mathcal{F}}\right)_{\mathbf{0}}^{\mathbf{2}}, t\right)=\sum_{\mathcal{G} \in M(\mathcal{F})} t^{\sum_{F \in \mathcal{G}}(|F|-2)+1} \prod_{F \in \mathcal{G}} f_{\mathbb{K}}\left(\Delta\left(M(\mathcal{F})_{\prec F}\right), t^{-1}\right) .
$$

Equation (19) can also be obtained from the general Goresky-MacPherson formula for the homology of subspace arrangements [8].

\subsection{The $(s, \mathbf{k})$-Equal Path Space}

Let $1 \leq s \leq n$ and $\mathbf{k}=\left(k_{1}, \ldots, k_{n}\right) \in \mathbb{N}_{+}^{n}$. The $(s, \mathbf{k})$-equal path space is defined as $\vec{P}\left(X_{\mathcal{F}_{n, s}}\right)_{\mathbf{0}}^{\mathbf{k}+\mathbf{1}}$ where $\mathcal{F}_{n, s}=\{F \subset[n]:|F| \geq s\}$. This path space occurs when every process $T_{i}$ calls upon a single resource a of capacity $s-1$ a number $k_{i}$ of times.

We use Formula (5) to obtain some information on the homology of this space. For $m \geq s$, let $\bar{\Pi}_{m, s}$ denote the poset of nontrivial partitions of $[m]$ such that every nonsingleton block has cardinality at least $s$. The homology of the order complex $\Delta\left(\bar{\Pi}_{m, s}\right)$ had been determined by Björner and Welker [3] and was further studied in [2, 10]. We will need the following result: 
Theorem 5.1 (Theorem 4.5 in [3], Corollary 6.2 in [2]). $\Delta\left(\bar{\Pi}_{m, s}\right)$ has the homotopy type of a wedge of spheres. The $d$-th Betti number of $\Delta\left(\bar{\Pi}_{m, s}\right)$ is nonzero iff $d=m-3-\ell(s-2)$ for some $1 \leq \ell \leq\left\lfloor\frac{n}{s}\right\rfloor$, and

$$
\tilde{\beta}_{m-3-\ell(s-2)}\left(\Delta\left(\bar{\Pi}_{m, s}\right)\right)=\sum_{\substack{j_{1}+\cdots+j_{\ell}=m \\
j_{i} \geq s}}\left(\begin{array}{c}
m-1 \\
j_{1}-1, j_{2}, \ldots, j_{\ell}
\end{array}\right) \prod_{i=0}^{\ell-1}\left(\begin{array}{c}
j_{i}-1 \\
s-1
\end{array}\right) .
$$

Note that $\Delta\left(M\left(\left(\mathcal{F}_{n, s}\right)_{\prec F}\right)\right) \cong \Delta\left(\bar{\Pi}_{|F|, s}\right)$ for any $F \in \mathcal{F}_{n, s}$. Theorem 1.3(i) implies that $H_{*}\left(\vec{P}\left(X_{\mathcal{F}_{n, s}}\right)_{0}^{\mathbf{k}+\mathbf{1}}\right)$ is free. Moreover, by Theorem 1.3(ii)

$$
\begin{aligned}
& f_{\mathbb{K}}\left(\vec{P}\left(X_{\mathcal{F}_{n, s}}\right)^{\mathbf{k}+\mathbf{1}}, t\right) \\
& =\sum_{0 \neq \mathbf{m} \in \mathbb{N}^{\mathcal{F}_{n, s}}} b_{\mathcal{F}, \mathbf{k}}(\mathbf{m}) t^{\mathcal{C}_{\mathcal{F}_{n, s}}(\mathbf{m})} \prod_{F \in \mathcal{F}_{n, s}} f_{\mathbb{K}}\left(\Delta\left(M\left(\left(\mathcal{F}_{n, s}\right)_{\prec F}\right)\right), t^{-1}\right)^{\mathbf{m}(F)} \\
& =\sum_{0 \neq \mathbf{m} \in \mathbb{N}^{\mathcal{F}_{n, s}}} b_{\mathcal{F}, \mathbf{k}}(\mathbf{m}) t^{\sum_{F \in \mathcal{F}_{n, s}} \mathbf{m}(F)(|F|-2)+1} \mu(\mathbf{m}, t)
\end{aligned}
$$

where

$$
\mu(\mathbf{m}, t)=\prod_{F \in \mathcal{F}_{n, s}}\left(\sum_{\ell=1}^{\left\lfloor\frac{\lfloor F \mid}{s}\right\rfloor} \tilde{\beta}_{|F|-3-\ell(s-2)}\left(\Delta\left(\bar{\Pi}_{|F|, s}\right)\right) t^{-|F|+2+\ell(s-2)}\right)^{\mathbf{m}(F)} .
$$

It follows that $t^{\alpha}$ appears in $f_{\mathbb{K}}\left(\vec{P}\left(X_{\mathcal{F}_{n, s}}\right)_{\mathbf{0}}^{\mathbf{k}+\mathbf{1}}, t\right)$ with nonzero coefficient only if $\alpha \equiv$ $1(\bmod (s-2))$.

Corollary 5.2. $\tilde{H}_{\ell}\left(\vec{P}\left(X_{\mathcal{F}_{n, s}}\right)_{0}^{\mathbf{k}+1} ; \mathbb{Z}\right)=0$ unless $\ell=m(s-2)$ for some $m>0$.

\subsection{The Connectivity of Path Spaces}

The following result determines the homological connectivity of $\vec{P}\left(X_{\mathcal{F}}\right)_{\mathbf{0}}^{\mathbf{k}+\mathbf{1}}$.

Proposition 5.3. Let $s(\mathcal{F})=\min _{F \in \mathcal{F}}|F|$. Then

$$
\min \left\{i: \tilde{H}_{i}\left(\vec{P}\left(X_{\mathcal{F}}\right)_{\mathbf{0}}^{\mathbf{k}+1} ; \mathbb{Z}\right) \neq 0\right\}=s(\mathcal{F})-2 .
$$

Proof: Choose an $F \in \mathcal{F}$ such that $|F|=s(\mathcal{F})$. Then $\Delta\left(M(\mathcal{F})_{\prec F}\right)$ is the empty complex $\{\emptyset\}$ and therefore $f_{\mathbb{K}}\left(\Delta\left(M(\mathcal{F})_{\prec F}\right), t^{-1}\right)=1$. Letting $\mathbf{m}\left(F^{\prime}\right)=1$ if $F=F^{\prime}$ and zero otherwise, it follows from Theorem 1.3(ii) that $t^{c_{\mathcal{F}}(\mathbf{m})}=t^{|F|-1}=t^{s(\mathcal{F})-1}$ appears in $f_{\mathbb{K}}\left(\vec{P}\left(X_{\mathcal{F}}\right)_{0}^{\mathbf{k}+1}, t\right)$ with a positive coefficient, and therefore $\tilde{H}_{s(\mathcal{F})-2}\left(\vec{P}\left(X_{\mathcal{F}}\right)_{0}^{\mathbf{k}+1} ; \mathbb{K}\right) \neq 0$.

For the other direction, first note that for any $F \in \mathcal{F}$

$$
\operatorname{dim} \Delta\left(M(\mathcal{F})_{\prec F}\right) \leq|F|-s(\mathcal{F})-1 .
$$


Therefore for any $F_{1}, \ldots, F_{r} \in \mathcal{F}$

$$
\begin{aligned}
\operatorname{dim} \underset{i=1}{*} \Delta\left(M(\mathcal{F})_{\prec F_{i}}\right) & \leq \sum_{i=1}^{r}\left(\left|F_{i}\right|-s(\mathcal{F})-1\right)+r-1 \\
& =\sum_{i=1}^{r}\left|F_{i}\right|-r s(\mathcal{F})-1 \\
& <\sum_{i=1}^{r}\left(\left|F_{i}\right|-2\right)-s(\mathcal{F})+2 .
\end{aligned}
$$

Thus

$$
\tilde{H}^{j}\left(S^{N-\sum_{i=1}^{r}\left(\left|F_{i}\right|-2\right)-1} * \underset{i=1}{*} \Delta\left(M(\mathcal{F})_{\prec F_{i}}\right)=0\right.
$$

for all

$$
\begin{aligned}
j & \geq\left(N-\sum_{i=1}^{r}\left(\left|F_{i}\right|-2\right)-1\right)+\left(\sum_{i=1}^{r}\left(\left|F_{i}\right|-2\right)-s(\mathcal{F})+2\right)+1 \\
& =N-s(\mathcal{F})+2 .
\end{aligned}
$$

As $\widehat{E_{\mathcal{F}}}$ is a wedge of spaces of the form

$$
S^{N-\sum_{i=1}^{r}\left(\left|F_{i}\right|-2\right)-1} * \underset{i=1}{*} \Delta\left(M(\mathcal{F})_{\prec F_{i}}\right)
$$

where $F_{1}, \ldots, F_{r} \in \mathcal{F}$, it follows that $\tilde{H}^{j}\left(\widehat{E_{\mathcal{F}}} ; \mathbb{Z}\right)=0$ for all $j \geq N-s(\mathcal{F})+2$. Finally, Alexander duality $\tilde{H}_{i}\left(\vec{P}\left(X_{\mathcal{F}}\right)_{\mathbf{0}}^{\mathbf{k}+\mathbf{1}} ; \mathbb{Z}\right) \cong \tilde{H}^{N-i-1}\left(\widehat{E_{\mathcal{F}}}\right)$ implies that $\tilde{H}_{i}\left(\vec{P}\left(X_{\mathcal{F}}\right)_{\mathbf{0}}^{\mathbf{k}+\mathbf{1}} ; \mathbb{Z}\right)=0$ for all $i \leq s(\mathcal{F})-3$.

In fact, we establish the following stronger result:

Proposition 5.4. Let $\mathbf{p}$ denote any directed path in $\vec{P}\left(X_{\mathcal{F}}\right)_{\mathbf{0}}^{\mathbf{k}+\mathbf{1}}$. Then $\pi_{i}\left(\vec{P}\left(X_{\mathcal{F}}\right)_{\mathbf{0}}^{\mathbf{k}+\mathbf{1}} ; \mathbf{p}\right)=0$ for all $i \leq s(\mathcal{F})-3$.

Proof. According to Proposition 2.2, we may replace $\vec{P}\left(X_{\mathcal{F}}\right)_{0}^{\mathbf{k}+1}$ with the homotopy equivalent space $D_{\mathcal{F}} \subset \stackrel{\circ}{\mathrm{k}}_{\mathrm{k}}$. Proposition 5.3 tells us that $D_{\mathcal{F}}$ is connected; hence we can choose any base point $\mathbf{p} \in D_{\mathcal{F}}$ in the following. Connectedness can also be concluded from the subsequent argument in the case $i=0$.

Let $F: S^{i} \rightarrow D_{\mathcal{F}}$ denote any continuous map. Its image $F\left(S^{i}\right)$ is compact and has thus positive distance from the compact set $\overline{E_{\mathcal{F}}} \subset \Delta_{\mathbf{k}} . F$ admits a smooth approximation $\tilde{F}: S^{i} \rightarrow \triangle_{\mathrm{k}}$ homotopic to $F$ and so close to $F$ that the image of the homotopy does not intersect $E_{\mathcal{F}}$. Extend $\tilde{F}$ to a smooth map $G: D^{i+1} \rightarrow \stackrel{\Delta}{\mathrm{k}}_{\mathrm{k}}$ by defining $G(0)=\mathbf{p}$ and by convex combination with $\tilde{F}$ on the boundary $S^{i}$. The image $G\left(D^{i+1}\right)$ may intersect $E_{\mathcal{F}}$. 
By multiple application of the transversality theorem (see e.g. [9, Theorem III.2.1],[1, Ch. I.2]), one can find a smooth approximation $H$ to $G$ that is transversal to all strata in $E_{\mathcal{F}}$. Moreover, since the compact sets $G\left(D^{i+1}\right)$ and $\partial \Delta_{\mathrm{k}}$ have a positive distance, we may assume that $H\left(D^{i+1}\right)$ is contained in $\Delta_{\mathbf{k}}$, as well. Each of the subspaces $G_{F, \mathbf{j}}$ in the definition of $E_{\mathcal{F}}$ has codimension $|F|-1$ in $\mathbf{R}^{N}$, and intersections have higher codimensions. In particular, if $i+1<|F|-1$, then $H\left(D^{i+1}\right) \cap G_{F, \mathbf{j}}=\emptyset$ by transversality. If $i+1<s(\mathcal{F})-1$, then $H\left(D^{i+1}\right) \cap E_{\mathcal{F}}=\emptyset$ and $H$ establishes that $\tilde{F}$ and hence $F$ are nul-homotopic in $D_{\mathcal{F}}$.

\section{Concluding Remarks}

We conclude with a few remarks about possible extensions of the results of this paper that we hope to deal with in future work. One obvious challenge concerns finding maps from spheres, and more generally products of spheres, into path space such that the images of the fundamental classes may serve as generators for homology in the appropriate dimensions, aiming at a generalization of [14, Corollary 3.10] in the paper of Raussen and Ziemiański. This is work in progress.

On the other hand, the situation we analysed is perhaps characterized by more regularity than what is needed for the method to work. The paper of Raussen and Ziemiański [14] calculates the homology of the path space $\vec{P}(X)_{0}^{\mathbf{k}+1}$ with $X=\mathbb{R}^{n} \backslash Y$ with $Y$ a subset of $\mathbb{Z}^{n}$. It seems likely that it is possible to extend our results to the following more general situation (with $\mathcal{F}$ an upward closed hypergraph on $[n]$ as previously):

For $F \in \mathcal{F}$ and $\alpha: F \rightarrow \mathbb{Z}$ a function, let $Y_{\alpha}:=\left\{\left(x_{1}, \ldots, x_{n}\right) \mid x_{i}=\alpha(i), i \in F\right\}$. For any non-empty subset $\beta(F) \subset \mathbb{Z}^{F}$ let $Y_{\beta(F)}:=\bigcup_{\alpha \in \beta(F)} Y_{\alpha}$. In the present paper, we only considered $\beta(F)=\mathbb{Z}^{F}$.

Now we assume that for every $F \in \mathcal{F}$ such a subset $\beta(F)$ has been chosen. Coherence suggests either to make a choice only for minimal elements of the family or to ask that $\beta\left(F_{2}\right)$ consists of all extensions of functions in $\beta\left(F_{1}\right)$ to $F_{2}$ in case $F_{1} \subset F_{2}$. The set to be excluded is then the union of hyperplanes $Y=\bigcup_{F \in \mathcal{F}} Y_{\beta(F)}$. It seems likely that one can determine the homology of $\vec{P}(X)_{\mathbf{0}}^{\mathbf{k}+\mathbf{1}}$ with $X=\mathbb{R}^{n} \backslash Y$, as well.

It is less obvious how to analyse topological properties of path spaces associated to general PV spaces (cf. Section 1) via arrangements - those would no longer be given by restrictions of linear subspaces. Instead, one has to remove thickened subspace arrangements within products of simplices leading to pattern spaces that are more difficult to analyse. For such thickened arrangements, our method - that makes essential use of the Wedge Lemma - is in general no longer applicable.

Since Ziemiański has shown [19] that every finite simplicial complex can arise as a connected component of the path space for some PV-space, one cannot expect a simple algorithmic determination of the homology of such a path space in general. 


\section{References}

[1] V.I. Arnold and S.M. Gusein-Zade and A.N. Varchenko, Singularities of Differentiable Maps, Volume 1, Basel: Birkhäuser, 1985.

[2] A. Björner and M. L. Wachs, Shellable nonpure complexes and posets. I, Trans. Amer. Math. Soc. 348(1996), 1299-1327.

[3] A. Björner and V. Welker, The homology of " $k$-equal" manifolds and related partition lattices, Adv. Math. 110(1995), 277-313.

[4] E.W. Dijkstra, Co-operating sequential processes, Programming Languages (F. Genuys, ed.), Academic Press, New York, 1968, 43-110.

[5] L. Fajstrup, É. Goubault, E. Haucourt, S. Mimram and M. Raussen, Directed Algebraic Topology and Concurrency, Springer-Verlag, Berlin, 2016.

[6] L. Fajstrup, É. Goubault, and M. Raussen, Algebraic Topology and Concurrency, Theor. Comput. Sci 357(2006), 241-278, Revised version of Aalborg University preprint, 1999.

[7] R. van Glabbeek, On the Expressiveness of Higher Dimensional Automata, Theor. Comput. Sci. 368(2006), 168-194.

[8] M. Goresky and R. MacPherson, Stratified Morse theory, Springer-Verlag, Berlin, 1988.

[9] M. Hirsch, Differential Topology, Springer-Verlag, New York, 1976.

[10] I. Peeva, V. Reiner and V. Welker, Cohomology of real diagonal subspace arrangements via resolutions, Compositio Math. 117(1999), 99-115.

[11] V. Pratt, Modelling concurrency with geometry, Proc. of the 18th ACM Symposium on Principles of Programming Languages. (1991), 311-322.

[12] M. Raussen, Simplicial models for trace spaces, Algebr. Geom. Topol. 10(2010), 16831714.

[13] M. Raussen, Simplicial models for trace spaces II: General higher-dimensional automata, Algebr. Geom. Topol. 12(2012), 1745-1765.

[14] M. Raussen and K. Ziemiański, Homology of spaces of directed paths on Euclidean cubical complexes, J. Homotopy Relat. Struct. 9(2014), 67-84.

[15] R. P. Stanley, Acyclic orientations of graphs, Discrete Math. 5(1973), 171-178.

[16] M. L. Wachs, Poset topology: tools and applications, in Geometric combinatorics, 497-615, IAS/Park City Math. Ser., 13, Amer. Math. Soc., Providence, RI, 2007. 
[17] J.W. Walker, Canonical homeomorphisms of posets, European J. Combin. 9(1988), 97-107.

[18] G. Ziegler and R. Živaljević, Homotopy types of subspace arrangements via diagrams of spaces, Math. Ann. 295(1993), 527-548.

[19] K. Ziemiański, On execution spaces of PV-programs, Theoret. Comput. Sci., 619(2016) 87-Ú98. 